\title{
A New Algorithm for Internet IP Level Topology Measurement
}

\author{
Shaolei Wang ${ }^{\mathrm{a}}$, Lei Zhang ${ }^{\mathrm{b}}$ and Chaojing Tang ${ }^{\mathrm{c}}$ \\ College of Electronic Science and Engineering, National University of Defense Technology, \\ Changsha, People's Republic of China

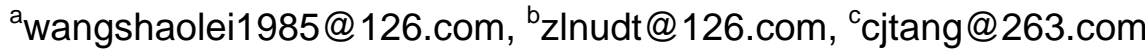

\begin{abstract}
Keywords: topology measurement; traceroute; load balancing; link inference
\end{abstract}
\begin{abstract}
Load balancing is one major cause of the false traceroute link problem in the Internet topology measurement field. In order to improve link inference correctness in the presence of load balancing, the impact of per-packet load balancing to link inference in the context of symmetric and asymmetric load balanced paths is analyzed. After that, a new algorithm for Internet IP level topology measurement is proposed. To evaluate the effectiveness of proposed algorithm, two experimental networks of symmetric and asymmetric per-packet load balanced paths are built. The corresponding measurement results of proposed algorithm are compared with classic traceroute and Paris traceroute. The evaluation results show that the proposed algorithm could improve the link inference correctness in the presence of per-packet load balancing.
\end{abstract}

\section{Introduction}

Traceroute is the most widely used tool for Internet IP interface level topology measurement. However, traceroute has various limitations [1-11], such as anonymous router, hidden router, unidirectional, load balancing, routing changes, etc. These limitations have led to the present incomplete and inaccurate Internet IP interface level topology. Among the limitations of traceroute, load balancing is one major cause of the false links in Internet topology measurements.

Traceroute assumes only one single path from probing source to probing destination. However, load balancing breaks this assumption. Load balancing is the capability of a router to distribute traffic over all the router network ports that have the same distance to the destination. Load balancing routers use three different algorithms to split packets on outgoing links: per-destination, which forwards all packets destined for a host to the same output interface; per-flow, which uses the same output interface for all packets that have the same flow identifier; per-packet, which makes forwarding decisions independently for each packet. Load balancing could lead to probe packets set with different TTL values taking different travel paths, and result in false traceroute links.

Sherwood et al. $[12,13]$ proposed to use the IP record route option to trace through load balancing routers. The major limitation of the IP record route option is that it can only record the first nine hops of a trace at most. Furthermore, probe packets enabled with the IP record route option are liable to be dropped or filtered by intermediate routers for security reasons. Augustin et al. [14-16] proposed a method of controlling the travel paths of probe packets by setting the flow identifiers in the packet headers, which could largely improve the link inference correctness in principle in the context of per-flow load balancing. However, their method still could not deal with link inferences in the context of per-packet load balancing.

In this paper, we propose a new algorithm for Internet topology measurement in the context of per-packet load balancing. At first, the impacts of per-packet load balancing to the link inferences in the context of symmetric and asymmetric load balanced paths are presented and analyzed. After that, a new algorithm for Internet topology measurement in the context of per-packet load balancing is proposed. Then, to evaluate the effectiveness of proposed algorithm in the context of per-packet load balancing, two experimental networks were constructed by GNS3 to simulate the cases of symmetric and asymmetric load balanced paths. The experiment results show that proposed algorithm could improve the link inference correctness in the context of per-packet load balancing. In the end, we summarize the work of this paper. 


\section{Problem}

There are two types of per-packet load balancing: equal cost path, applicable when different paths to a destination network report the same routing metric value; unequal cost path, applicable when different paths to a destination network report are of different routing metric values $[17,18]$. As a consequence, there are two types of per-packet load balanced paths: symmetric paths and asymmetric paths. In this section, traceroute link inference in the context of symmetric per-packet load balanced paths and asymmetric per-packet load balanced paths is analyzed separately.

Symmetric per-packet load balanced paths. An example of tracerouting in the context of symmetric per-packet load balanced paths is shown in Fig. 1. $S$ is the probing source, routers $L, A$ and $C$ are all configured with equal cost path per-packet load balancing that make forwarding decisions independently for each packet. All possible false links during traceroute measurement processes in this case are shown in Fig. 2.

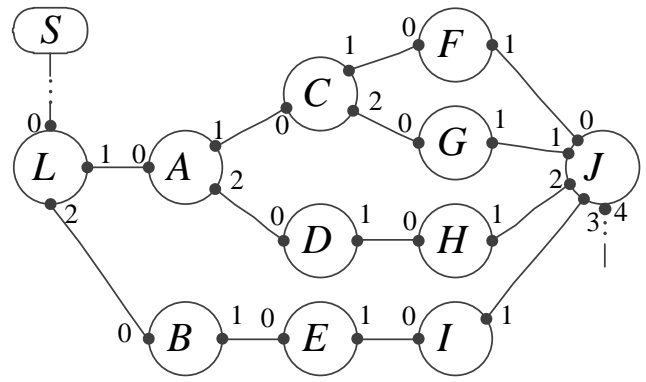

Fig. 1 The topology of symmetric per-packet load balanced paths

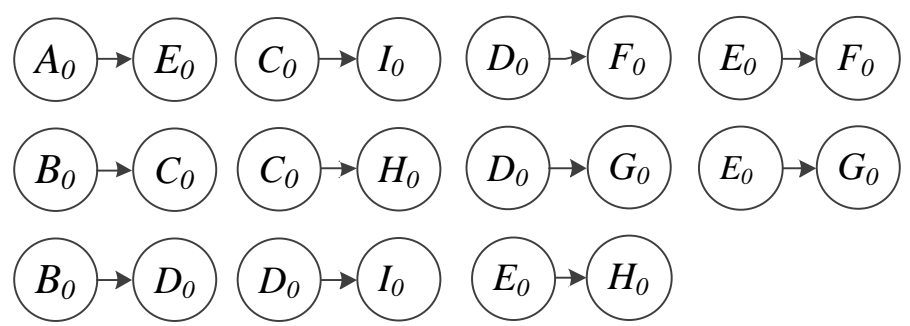

Fig. 2 False links caused by symmetric per-packet load balanced paths

Actually, in the context of symmetric per-packet load balanced paths, the response addresses of probe packets which are set with some special TTL value during traceroute measurement processes will change randomly among the node addresses that have the corresponding distance from the probing source. As a consequence, the links of adjacent hop addresses along the load balanced paths will also change randomly, which will cause a large number of false link inferences.

Asymmetric per-packet load balanced paths. An example of tracerouting in the context of asymmetric per-packet load balanced paths is shown in Fig. 3. $S$ is the probe source, router $L$ is configured with unequal cost path per-packet load balancing, router $A$ and $C$ are both configured with equal cost path per-packet load balancing, $L, A$ and $C$ all make forwarding decisions independently for each packet. All possible false links during traceroute measurement processes in this case are shown in Fig. 4.

Actually, in the context of asymmetric per-packet load balanced paths, the response addresses of probe packets which are set with some special TTL value during traceroute measurement processes will also change randomly among the node addresses that have the corresponding distance from the probing source. Furthermore, the number of node addresses that have the corresponding distance from the probing source will be much larger than that of the symmetric per-packet load balanced paths due to the path asymmetries. As a consequence, the links of adjacent hop addresses along the load balanced paths will change more randomly, which will cause much more false link inferences than the symmetric per-packet load balancing case. 


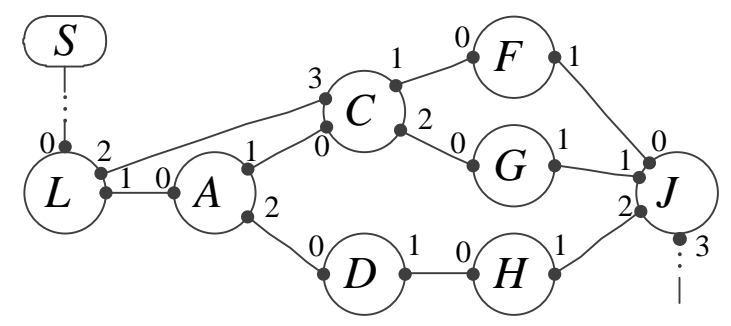

Fig. 3 The topology of asymmetric per-packet load balanced paths

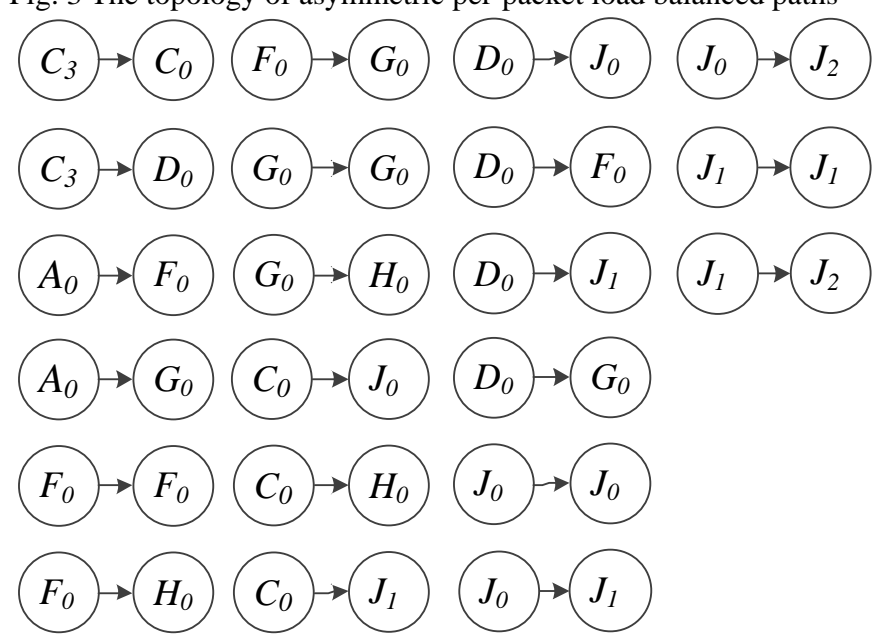

Fig. 4 False links caused by asymmetric per-packet load balanced paths

As we can see from the above examples, the false link inferences of traceroute in the context of per-packet load balancing is mainly caused by the random travel paths of probe packets set with special TTL values. Furthermore, as is limited by the traceroute measurement principle, each probe packet's actual travel path will be determined by the routers along its possible travel paths once it is set and sent out. As a consequence, there may be many false link inferences when tracerouting in the context of per-packet load balancing.

\section{Algorithm}

To improve the link inference correctness of tracerouting in the context of per-packet load balancing, a new topology measurement algorithm is proposed. The main process of proposed algorithm is shown in Fig. 5.

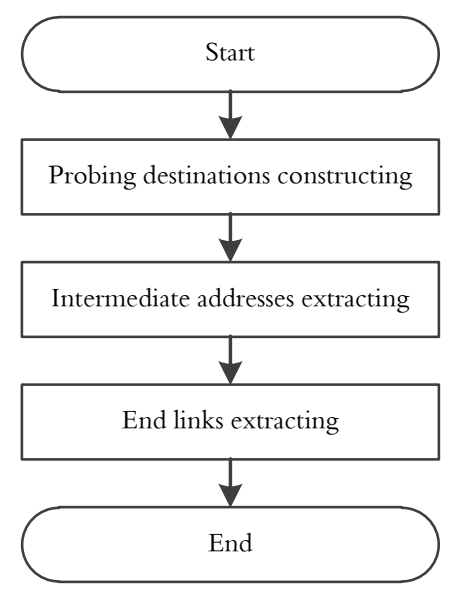

Fig. 5 Flow chart of proposed algorithm

Probing destinations constructing. This is the first part of proposed algorithm, the main function of which is to construct the particular probing destination address set according to the specific topology measurement mission. To the Internet topology measurement, constructing probing destination addresses including determining the measurement scope, selecting the measurement granularity and designing the probing destinations constructing policy. 
Intermediate addresses extracting. The main function of this part is to extract the intermediate router interface address set from probing source to probing destinations by common traceroute. The router interface addresses acquired by traceroute in the Internet environment may include private addresses. These private addresses will be filtered by pre-established rules and only the rest public addresses will be recorded and passed on to the next processing part.

End links extracting. This part is the core of proposed algorithm. Each public address acquired by former intermediate addresses extracting part will be set as a new probing destination. After that, these new probing destinations will be tracerouted and the resulting end links will be used to construct the Internet IP level topology.

Denote the intermediate address set as $N_{m}=\left\{n_{i} \mid 0<=i<=P\right\}$. Then, each special intermediate address $n_{i}$ will be set as a new probing destination. The probing process is circular. In each circle, the probe packets set with incremental TTL value will be sent out until they reach $n_{i}$. The control policy of this circular process is similar to the method of Paris traceroute [15]. When this circular probing process to some special address $n_{i}$ is finished, extracting the end address set before $n_{i}$ is reached. Denote this end address set as $S_{i}=\left\{n_{u} \mid 0<=u<=Q\right\}$, then $\left\{\left(n_{u}, n_{i}\right) \mid 0<=u<=Q\right\}$ is the end link set of $n_{i}$. Also, if some special address $n_{u}$ does not exist in $N_{m}$, it will be added to $N_{m}$. If some special address $n_{i}$ is not responsive to probing, then it will be filtered. In the end, each address in $N_{m}$ will be probed and the end links extracting process will be finished. The resulting end link sets will be the topology measurement results.

\section{Evaluation}

To evaluate the effectiveness of proposed algorithm in the context of per-packet load balancing, two experimental networks were constructed by GNS3 to simulate the cases of symmetric and asymmetric load balanced paths. Then, topology measurement experiments were carried out by using traditional traceroute, Paris traceroute and proposed algorithm. After that, the measurement results were compared and analyzed.

Symmetric load balanced paths. The network topology of the symmetric per-packet load balanced paths case is shown in Fig. 6. Router $L$ was configured with even cost per-packet load balancing. In the measurement process, the probing source was connected to interface $L_{0}$ and probing destinations were set with IP addresses of subnets connected with $J_{4}$. Specifically, the probing destination of Paris traceroute was some single IP address chosen randomly, the probing destinations of traditional traceroute and proposed algorithm were 10 IP addresses chosen randomly.

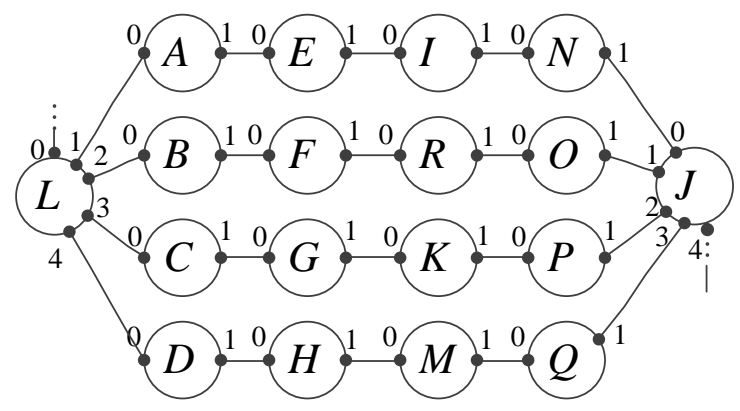

Fig. 6 The topology of symmetric load balanced paths

The measurement results are shown in Table. 1 . As we can see from the topology of experimental network, there are 20 distinct actual physical links in all. Traditional traceroute discovered 14 distinct links in all, only 5 links were verified as correct and corresponding to 5 distinct actual physical links, the link inference correctness of which is $35.7 \%$. Paris traceoute discovered 68 distinct links in all, only 32 links were verified as correct and corresponding to 20 distinct actual physical links, the link inference correctness of which is $47.1 \%$. The proposed algorithm discovered 20 distinct links in all, all the 20 links were verified as correct and corresponding to 20 distinct actual physical links, the link inference correctness of which is $100 \%$. 
Tab. 1 Measurement results of symmetric load balanced paths

\begin{tabular}{llll}
\hline Measurement results & tradional traceroute & Paris traceroute & proposed algorithm \\
\hline Total links & 14 & 68 & 20 \\
Correct links & 5 & 32 & 20 \\
Physical links & 5 & 20 & 20 \\
Inference correctness & $35.7 \%$ & $47.1 \%$ & $100 \%$ \\
\hline
\end{tabular}

Asymmetric load balanced paths. The network topology of the asymmetric per-packet load balanced paths case is shown in Fig. 7. Router $L$ was configured with uneven cost per-packet load balancing to construct asymmetric load balanced paths. The measurement process is similar to the case of symmetric load balanced paths.

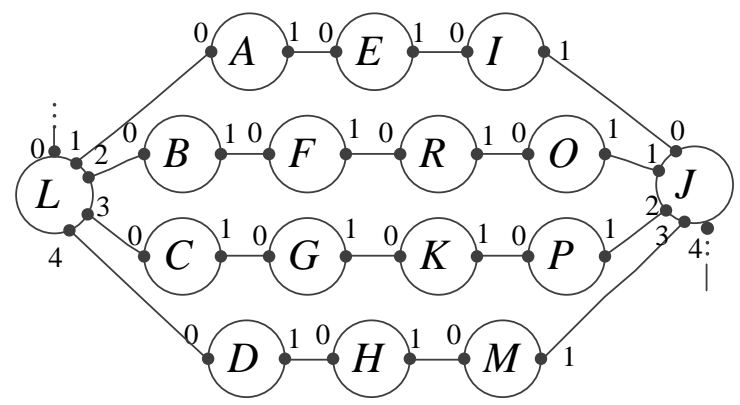

Fig. 7 The topology of asymmetric load balanced paths

The measurement results are shown in Table. 2. As we can see from the topology of experimental network, there are 18 distinct actual physical links in all. Traditional traceroute discovered 13 distinct links in all, only 4 links were verified as correct and corresponding to 4 distinct actual physical links, the link inference correctness of which is $30.7 \%$. Paris traceoute discovered 60 distinct links in all, only 20 links were verified as correct and corresponding to 18 distinct actual physical links, the link inference correctness of which is $33.3 \%$. The proposed algorithm discovered 26 distinct links in all, all the 24 links were verified as correct and corresponding to 18 distinct actual physical links, the link inference correctness of which is $92.3 \%$.

Tab. 2 Measurement results of asymmetric load balanced paths

\begin{tabular}{llll}
\hline Measurement results & tradional traceroute & Paris traceroute & proposed algorithm \\
& & & \\
\hline Total links & 13 & 60 & 26 \\
Correct links & 4 & 20 & 24 \\
Physical links & 4 & 18 & 18 \\
Inference correctness & $30.7 \%$ & $33.3 \%$ & $92.3 \%$ \\
\hline
\end{tabular}

As we can see from the above measurement results, the link inference correctness of proposed algorithm, traditional traceroute and Paris traceroute all become lower than in the case of symmetric load balanced paths due to the extra randomness introduced by asymmetries. However, proposed algorithm's link inference correctness is still higher than traditional traceroute and Paris traceroute.

\section{Summary}

Load balancing is one major cause of the false traceroute link problem in the Internet topology measurement field. In this paper, the impact of per-packet load balancing to link inference in the context of symmetric and asymmetric load balanced paths is analyzed. After that, a new algorithm is proposed for Internet topology measurement in the context of per-packet load balancing. Then, experimental networks of symmetric and asymmetric load balanced paths were constructed. Topology measurement experiments were carried out by using proposed algorithm, traditional traceroute and Paris traceroute. The topology measurement experiment results show that proposed algorithm could improve the link inference correctness in the context of per-packet load balancing. 


\section{References}

[1] Valentini F., Pratesi M., Santucci F., et al. IPv4 and IPv6 troubleshooting enhancement through reverse path discovery; proceedings of the Network Operations and Management Symposium (NOMS), 2014 IEEE, F, 2014 [C]. IEEE.

[2] Prabhavat S., Nishiyama H., Ansari N., et al. On load distribution over multipath networks [J]. Communications Surveys \& Tutorials, IEEE, 2012, 14(3): 662-80.

[3] Krishnamurthy A., Singh A. Robust multi-source network tomography using selective probes; proceedings of the INFOCOM, 2012 Proceedings IEEE, F, 2012 [C]. IEEE.

[4] Schwartz Y., Shavitt Y., Weinsberg U. On the diversity, stability and symmetry of end-to-end Internet routes; proceedings of the INFOCOM IEEE Conference on Computer Communications Workshops, 2010, F, 2010 [C]. IEEE.

[5] Shih M.-F., Hero A. O. Hierarchical inference of unicast network topologies based on end-to-end measurements [J]. Signal Processing, IEEE Transactions on, 2007, 55(5): 1708-18.

[6] Donnet B, Friedman T. Internet topology discovery: a survey[J]. Communications Surveys \& Tutorials, IEEE, 2007, 9(4): 56-69.

[7] Donnet B. Internet topology discovery[M]//Data Traffic Monitoring and Analysis. Springer Berlin Heidelberg, 2013: 44-81.

[8] Marchetta P, Pescape A. Drago: Detecting, quantifying and locating hidden routers in traceroute IP paths[C]//INFOCOM, 2013 Proceedings IEEE. IEEE, 2013: 3237-3242.

[9] Luckie M, Dhamdhere A, Murrell D. Measured impact of crooked traceroute[J]. ACM SIGCOMM Computer Communication Review, 2011, 41(1): 14-21.

[10]He Y, Faloutsos M, Krishnamurthy S, et al. On routing asymmetry in the Internet[C]//Global Telecommunications Conference, 2005. GLOBECOM'05. IEEE. IEEE, 2005, 2: 6 pp.

[11]Gunes M H, Sarac K. Resolving anonymous routers in internet topology measurement studies[C]//INFOCOM 2008. The 27th Conference on Computer Communications. IEEE. IEEE, 2008.

[12] Sherwood R, Spring N. Touring the Internet in a TCP Sidecar[C]//Proceedings of the 6th ACM SIGCOMM conference on Internet measurement. ACM, 2006: 339-344.

[13] Sherwood R, Bender A, Spring N. Discarte: a disjunctive internet cartographer[C]//ACM SIGCOMM Computer Communication Review. ACM, 2008, 38(4): 303-314.

[14] Augustin B, Cuvellier X, Orgogozo B, et al. Avoiding traceroute anomalies with Paris traceroute[C]//Proceedings of the 6th ACM SIGCOMM conference on Internet measurement. ACM, 2006: 153-158.

[15]Augustin B, Friedman T, Teixeira R. Multipath tracing with Paris traceroute[C]//End-to-End Monitoring Techniques and Services, 2007. E2EMON'07. Workshop on. IEEE, 2007: 1-8.

[16] Augustin B, Friedman T, Teixeira R. Measuring multipath routing in the internet[J]. IEEE/ACM Transactions on Networking (TON), 2011, 19(3): 830-840.

[17]Cisco. How Does Load Work? http://www.cisco.com/c/en/us/support/docs/ip/border-gateway-protocol-bgp/5212-46.html

[18] Cisco. How Does Unequal Cost Path Load Balancing (Variance) Work in IGRP and EIGRP? http://www.cisco.com/c/en/us/support/docs/ip/enhanced-interior-gateway-routing-protocol-eigrp/13 677-19.html 